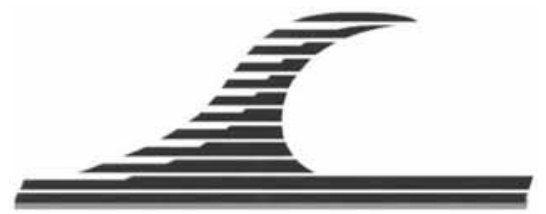

Mots-clés : Capacité de terminaux, Conteneurs, Quais,

Simulation.

(C) Editions Paralia CFL

\title{
Modélisation continue de l'accostage des navires pour le calcul de la capacité des quais. Application aux grands terminaux à conteneurs espagnols
}

\author{
Roser OBRER-MARCO ${ }^{1}$, José AGUILAR ${ }^{2}$
}

1. Universidad Politécnica de Valencia, Departamento de Ingeniería e Infraestructura de los Transportes, Camino de Vera s/n, 46.022 Valencia, Espagne.

roobmar@gmail.com;jaguilar@upv.es

\section{Résumé :}

Le Système Portuaire Espagnol (SPE) est composé de 46 ports d'intérêt général, gérés par 28 Autorités Portuaires. Les relations entre ces autorités portuaires sont de nature concurrentielle, ce qui fait de la planification un outil indispensable pour qu'elles soient compétitives. Grâce à la planification les gérants des ports sont capables d'ajuster la capacité et les conditions physiques des infrastructures portuaires aux prévisions de croissance du trafic.

Il est essentiel que les départements de planification des Autorités Portuaires espagnoles disposent d'un outil qui soit capable d'estimer la capacité des infrastructures, et plus précisément des quais. Jusqu’à présent, le plus courant pour estimer cette capacité a été d'appliquer une formulation qui assume un nombre déterminé de postes d'accostage, tous identiques: formulation discrète. Néanmoins, l'exploitation des quais des terminaux à conteneurs est faite de manière continue. Pour cette raison, il faut faire un pas en avant et utiliser une formulation qui reflète l'exploitation réelle des quais : formulation continue.

Dans cet article on présente une formulation continue et on la compare avec la formulation discrète, en mettant en évidence les erreurs qui peuvent être commises en supposant que le quai est composé d'un ensemble de postes d'accostage identiques. Pour cela, on a appliqué les deux formulations au terminal à conteneurs MSC Terminal Valencia, S.A. du port de Valencia (Espagne) avant et après son agrandissement en 2010.

Soumis le 20 janvier 2012, accepté le 16 juin 2014, en ligne le 15 décembre 2014. La seule version examinée est celle écrite en français. La ou les autres versions n'étant pas examinées par le comité de rédaction de la revue, sont donc publiées sous l'entière responsabilité du ou des auteurs.

UNA VERSION TRADUCIDA EN ESPAÑOL ES DISPONIBLE EN LINEA

Pour citer cet article :

OBRER-MARCO R., AGUILAR J. (2014). Modélisation continue de l'accostage des navires pour le calcul de la capacité des quais. Application aux grands terminaux à conteneurs espagnols. Revue Paralia, Vol. 7, pp n02.1-n02.12.

DOI: http://dx.doi.org/10.5150/revue-paralia.2014.n02 


\section{Introduction et objectifs}

La capacité d'un quai est le trafic limite qui peut être admis par le quai pendant une période de temps déterminée, normalement une année, mais, comme la définition de trafic limite n’est pas consensuelle, le concept de capacité ne peut pas être unique. On peut citer trois définitions de trafic limite (RODRIGUEZ, 1977): le trafic de congestion, trafic à partir duquel les attentes des navires commencent à augmenter très rapidement ; le trafic économique, trafic où l'addition des coûts des navires et des coûts du quai est minimale ; ou encore le trafic de saturation, trafic à partir duquel tous les postes d'accostage sont occupés en permanence.

Néanmoins, dans les grands terminaux à conteneurs et comme conséquence de la grande concurrence qui existe entre eux, ce trafic limite doit être lié au niveau de service offert aux clients du terminal, dans ce cas, les compagnies maritimes.

Jusqu'à maintenant, pour les terminaux à conteneurs, la formulation employée afin d'estimer le trafic considère que les quais sont composés d'un nombre déterminé de postes d'accostage identiques (formulation discrète).

Toutefois, dans la réalité, les quais des grands terminaux à conteneurs ne sont pas exploités de manière discrète, mais de manière continue. Par conséquent une formulation discrète implique le fait d'avoir réalisé une translation conceptuelle du quai : d'une ligne continue à un nombre de postes d'accostage $(N)$.

Le principal objectif de cet article est de présenter une formulation continue du calcul de la capacité des quais, afin de constater comment l'estimation de cette capacité peut changer quand celui-ci est modélisé comme un nombre déterminé de postes d'accostage ou, au contraire, comme une ligne continue. Le cas étudié pour illustrer cet objectif est celui de MSC Terminal Valencia, S.A. (MSCTV).

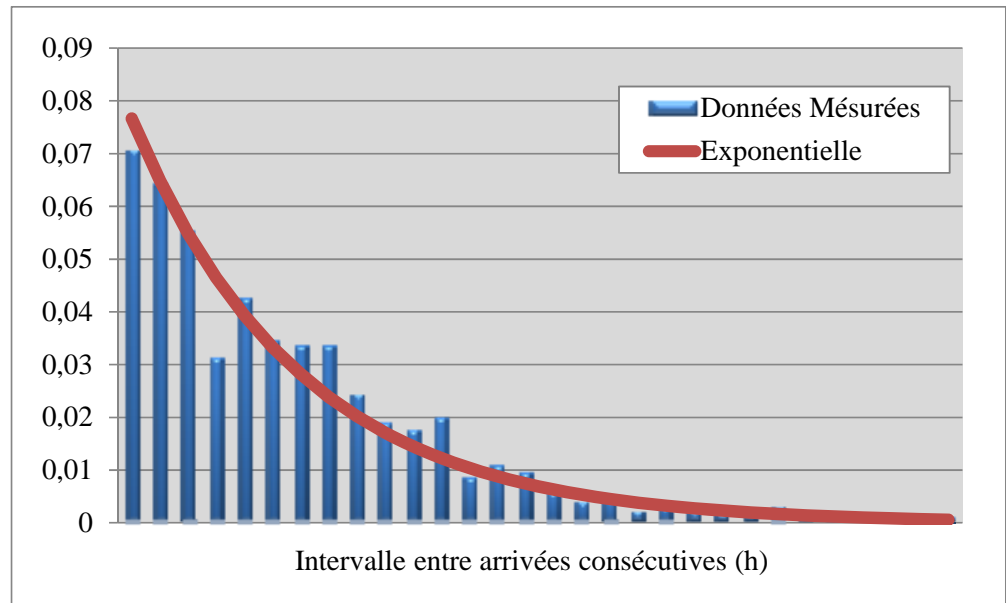

Figure 1. Densité de probabilité des arrivées des navires au terminal MSCTV.

Le terminal MSCTV a commencé ses opérations au Port de Valencia en 2007, et des 656 m opératifs initiaux, il est passé à 756 m en 2010. Pour le terminal MSCTV, avec 
des données entre janvier 2008 et juin 2009, la densité de probabilité des arrivées des navires est exponentielle (voir figure 1) et celle des durées de service est Erlang 5 (voir figure 2). La longueur moyenne des porte-conteneurs est d'environ $225 \mathrm{~m}$.

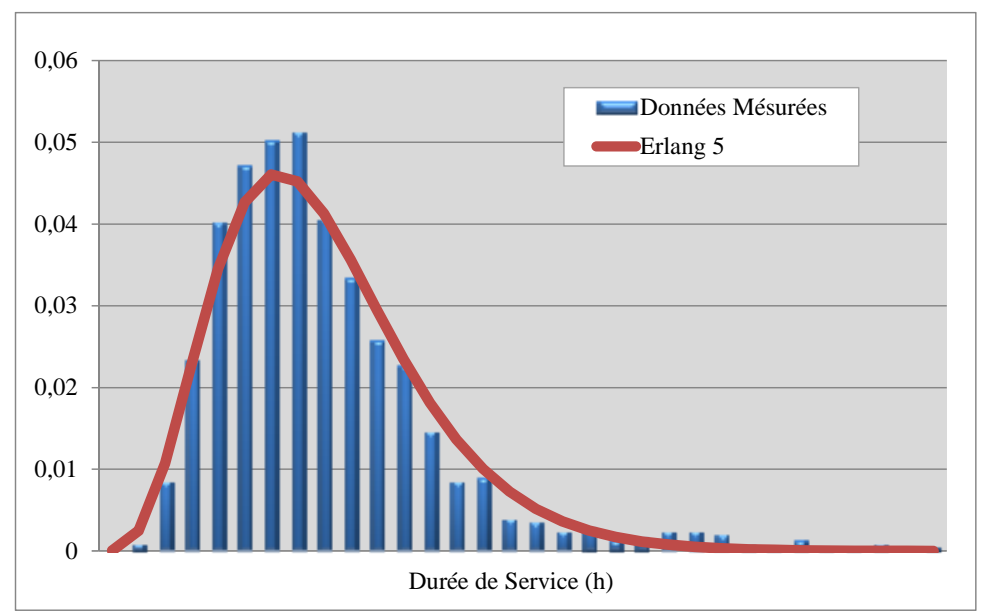

Figure 2. Densité de probabilité des durées du service au terminal MSCTV.

\section{La formulation discrète}

Une formulation possible et couramment utilisée pour estimer le trafic limite est :

$$
r=N \times \rho \times P \times H_{a n}
$$

où :

$r=$ trafic annuel du quai (conteneur Equivalent Vingt Pied ou EVP/an ou mouvements/an)

$N=$ nombre de postes d'accostage (en fonction de la longueur des navires, $N$ peut être un nombre décimal)

$\rho=$ taux d'occupation du quai (ou d'un poste d'accostage puisqu'ils coïncident)

$P=$ productivité du poste d'accostage (EVP/h ou mouvements/h)

$H_{a n}=$ heures opératives du terminal par an (h/an)

Le taux d'occupation $(\rho)$ est défini comme la proportion entre le temps où les postes d'accostage sont occupés et la totalité du temps où les postes sont disponibles (voir équation (5)).

Dans la formulation représentée par l'équation (1), il y a implicitement un niveau de service offert à la compagnie maritime à travers les variables $P$ et $\rho$. En ce qui concerne la capacité, l'intérêt des compagnies maritimes réside dans un temps d'escale réduit en proportion du nombre de conteneurs à manipuler pendant l'escale. Pour atteindre ce temps d'escale réduit il faut que la productivité du poste d'accostage $(P)$ soit élevée, et que les attentes au mouillage dues à la congestion soient réduites sinon inexistantes. En réalité, la productivité du poste d'accostage ainsi que l'attente apparaissent dans l'équation (1); la première directement et la deuxième indirectement au travers de $\rho$. Intuitivement, quand $\rho$ tend vers un (occupation permanente des postes d'accostage), les 
attentes des navires tendent vers l'infini, et de manière analogue, quand $\rho$ tend vers zéro, les attentes des navires sont pratiquement inexistantes.

En outre, il est raisonnable d'admettre que les attentes des navires sont proportionnelles à la durée de service. La relation entre l'attente moyenne des navires $(D a)$ et la durée de service (période de temps pendant laquelle le navire est amarré) moyenne de ces mêmes navires $(D s)$ est connue comme l'attente relative $\left(\varepsilon_{r}\right)$ :

$\varepsilon_{r}=\frac{D_{a}}{D_{S}}$

En connaissant la valeur de $N$, la densité de probabilité de l'intervalle entre arrivées consécutives des navires au terminal (A) et la densité de probabilité des durées de service au quai (B), il est possible de connaître la relation entre $\rho$ et $\varepsilon_{r}$ grâce à la théorie des files d'attente ou grâce à des outils de simulation.

La théorie des files d'attente ne peut être strictement appliquée que dans certains cas (RODRIGUEZ, 1977) :

1) Quand A et B sont des fonctions de répartition exponentielles, indépendamment de $N$.

2) Quand A ou B sont des fonctions de répartition exponentielles et $N=1$.

Dans tous les grands terminaux à conteneurs espagnols, on a vérifié que même si les arrivées des navires sont aléatoires, les durées de service suivent une densité de probabilité de la distribution d'Erlang $K$, où $K$ varie entre 4 et 7 (AGUILAR \& OBRER-MARCO, 2009). En conséquence, pour connaître la relation entre $\varepsilon_{r}$ et $\rho$ il est nécessaire d'utiliser des outils de simulation. La figure 3 montre une représentation de cette relation quand $\mathrm{A}$, B et $N$ sont déterminés.

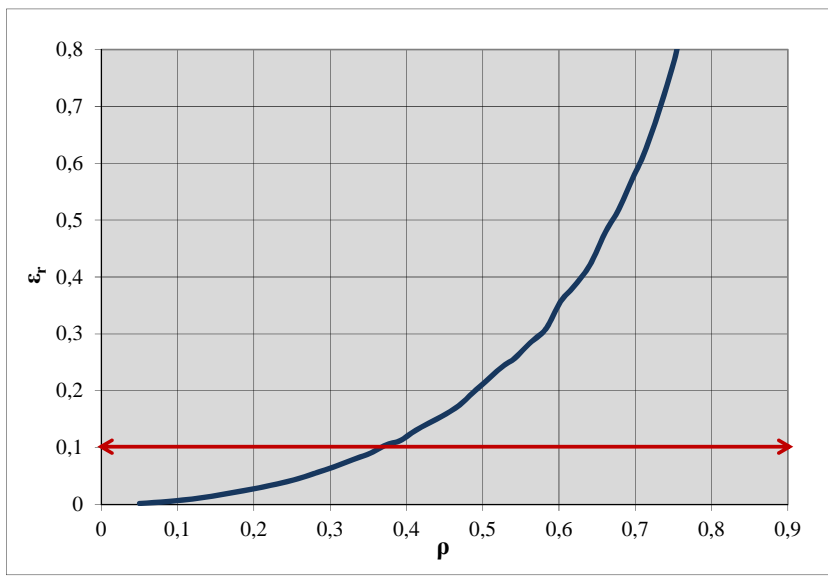

Figure 3. Exemple de représentation de la relation entre $\varepsilon_{r}$ et $\rho$ obtenue par simulation.

En supposant que la productivité du poste d'accostage $(P)$ est élevée, le niveau de service offert aux compagnies maritimes est représenté par $\varepsilon_{r}$. Quand ce niveau reste fixé à une situation limite admissible par les compagnies maritime, c'est-à-dire quand la 
relation entre la durée d'attente au mouillage et la durée de service au quai atteint une valeur maximale que les compagnies sont disposées à supporter, le trafic donné par la formulation équivaut à la capacité. Cette valeur maximale est la limite à partir de laquelle les compagnies maritimes partiraient solliciter escale dans d'autres terminaux. Alors, une fois la relation entre $\varepsilon_{r}$ et $\rho$ connue, il ne reste qu'à établir une valeur maximale de $\varepsilon_{r}$ pour connaître $\rho$. Dans le cas des terminaux à conteneurs une valeur de $\varepsilon_{r}$ supérieur à 0,1 n’est pas recommandée (AGERSCHOU, 2004).

\section{Méthodologie}

La méthodologie suivie pour mener les objectifs à bien fut la suivante. Tout d'abord, une nouvelle formulation du trafic du quai a été développée. Ensuite, les deux formulations existantes, celle qui suppose le quai composé d'un nombre $N$ de postes d'accostage (formulation discrète) et celle qui suppose le quai comme une ligne continue (formulation continue), ont été appliquées au quai de MSCTV avant et après son agrandissement en 2010. Finalement, les résultats des quatre calculs ont été comparés et des conclusions tirées.

Pour appliquer la formulation discrète sur un quai exploité de manière continue, $\mathrm{N}$ peut être obtenu en divisant la longueur du quai par la longueur moyenne des navires, laquelle doit être augmentée pour tenir compte d'une distance de sécurité entre les navires, voir l'équation (3). En pratique, cette augmentation vaut souvent $10 \%$ de la longueur moyenne des navires. La translation conceptuelle du quai peut supposer des erreurs dans l'estimation de la capacité.

$$
N=\frac{L}{1,10 \times L_{\text {navires }}}
$$

où :

$L=$ longueur du quai

$L_{\text {navires }}=$ longueur moyenne des navires qui font escale

\section{Formulation continue}

La capacité d'un quai conceptuellement exploité de manière continue peut être obtenue grâce à l'expression suivante :

$$
r=L \times \rho_{L} \times P_{L} \times H_{a n}
$$

où :

$r=$ trafic annuel du quai (EVP/an ou mouvements/an)

$L=$ longueur du quai (m)

$\rho_{L}=$ taux d'occupation du quai exploité de manière continue

$P_{L}=$ productivité du quai $(\mathrm{EVP} /(\mathrm{h} \cdot \mathrm{m})$ ou mouvements $/(\mathrm{h} \cdot \mathrm{m}))$

$H_{a n}=$ heures opératives du terminal par an (h/an) 
En comparant les formulations représentées par les expressions (1) et (4), on constate la similitude et l'équivalence entre leurs variables. Ainsi, $N$ et $L$ représentent la dimension du quai, $N$ de manière discrète et $L$ de manière continue. $P$ et $P_{L}$ représentent la vitesse de manipulation des conteneurs entre le quai et les navires, par unité de poste d'accostage $(P)$, ou de mètre de quai $\left(P_{L}\right)$; et $H_{a n}$ n'a aucune variation entre les deux formulations. Par contre, même si apparemment les variables $\rho$ et $\rho_{L}$ sont équivalentes, il $\mathrm{y}$ a une différence très importante dans leur définition et dans la manière de les estimer. En conséquence, la comparaison entre $\rho$ et $\rho_{L}$ doit se faire avec une extrême précaution (voir section suivante).

\section{Différence de calcul entre $\rho$ et $\rho_{L}$}

Afin de calculer la valeur de $\rho$ pendant une période de temps $T$, il est nécessaire de connaître la proportion entre la période de temps où les postes d'accostage sont occupés par un navire et la période de temps où les postes sont disponibles. Ainsi, le calcul de $\rho$ ne tient pas compte du fait que le navire occupe entièrement le poste ou non. Ce fait est illustré en comparant les deux situations présentées dans la figure 4, pour lesquelles les navires restent dans les deux cas accostés pendant une même période $\mathrm{T}$ à quelques jours de différence. Dans les deux situations, la valeur de $\rho$ est la même et égale à un, puisque dans les deux situations les deux postes d'accostage ont été occupé pendant la période complète de $T$. Néanmoins, la réalité est que dans la situation de droite les bateaux occupent complètement les postes, alors que dans la situation de gauche, les bateaux occupent seulement une faible partie des postes d'accostage. Ce phénomène n’a aucun impact dans les résultats de $\rho$.

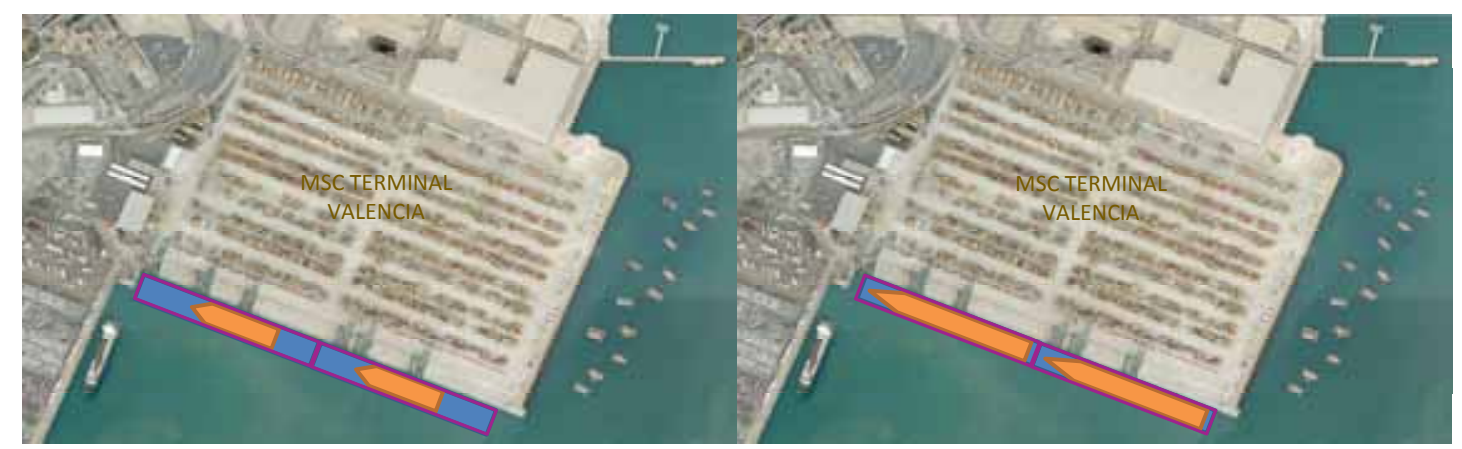

Figure 4. Deux situations différentes où $\rho$ a la même valeur.

Une formulation possible pour estimer $\rho$ est la suivante :

$\rho=\frac{\sum_{i=1}^{N} \frac{t_{i}}{T_{i}}}{N}$

où :

$\rho=$ taux d'occupation du quai 
$i=$ compteur de postes d'accostage

$N=$ nombre de postes d'accostage

$t_{i}=$ période de temps où le poste $i$ est occupé

$T_{i}=$ période de temps où le poste $i$ est disponible pour être occupé

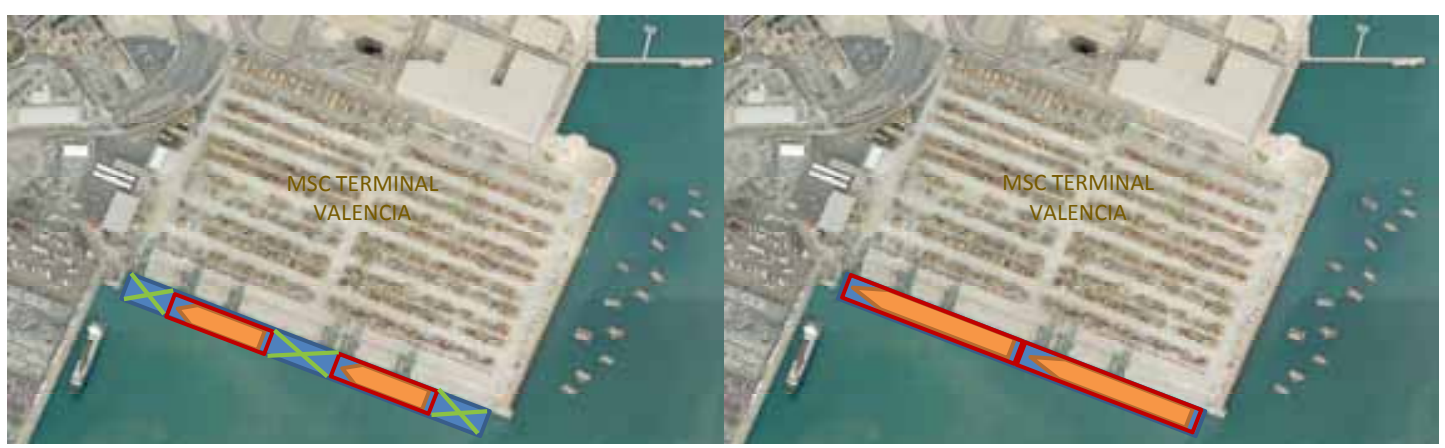

Figure 5. Deux situations différentes où $\rho_{L}$ a une valeur différente.

La figure 5 montre deux situations où les longueurs des navires sont exactement les mêmes que celles de la figure 4. La différence avec cette dernière est que le quai est conceptualisé comme une ligne continue et pas comme deux postes d'accostage.

Pour calculer la valeur de $\rho_{L}$ il faut prendre en compte la longueur des navires. Donc, même si les deux navires restent accostés pendant un même temps $T$ dans les deux situations de la figure 5 , la valeur de $\rho_{L}$ sera plus grande dans la situation droite. En effet, le nombre de mètres réellement occupés dans cette situation est plus grand.

La valeur de $\rho_{L}$ peut être calculée à partir de l'expression (6) :

$\rho_{L}=\frac{\sum_{j=1}^{v} 1,10 \times L_{\text {Navire } j} \times D_{S j}}{L \times T}$

où :

$j=$ compteur de navires

$v=$ nombre de navires qui font escale au terminal

$L_{\text {navires }}=$ =longueur du navire $j$

$D_{S j=}$ durée du service du navire $j$

$L=$ longueur du quai

$T=$ période de temps où le quai est disponible

La relation $\varepsilon_{r}-\rho$ (discrète) se calcule donc indépendamment de la longueur des navires au poste d'accostage, alors que, la relation $\varepsilon_{r}-\rho_{L}$ (continue) prend uniquement en compte les mètres occupés par le navire et une marge de sécurité (10\% de la longueur). En d'autres termes, $\rho_{L}$ symbolise un rapport entre des mètres-heures occupés et des mètres-heures totaux, d'où la différence essentielle entre les calculs de $\rho$ et $\rho_{L}$. 
Afin de connaître $\rho_{L}$ un programme de simulation basé sur des techniques de Monte Carlo a été développé. Il fournit la relation $\varepsilon_{r}-\rho_{L}$ à partir des donnés du terminal et des navires qui y font escale.

\section{Scénarios étudiés}

Pour mener les objectifs à bien, la capacité a été calculée selon quatre scénarios différents. Les deux premiers correspondent au quai de MSCTV avant son allongement et les deux derniers après. Les quatre scénarios sont :

1) quai de $656 \mathrm{~m}$ et formulation discrète ;

2) quai de $656 \mathrm{~m}$ et formulation continue ;

3) quai de $756 \mathrm{~m}$ et formulation discrète ;

4) quai de $756 \mathrm{~m}$ et formulation continue.

Dans tous les scénarios, la densité de probabilité de la longueur des navires est constante et égale à $225 \mathrm{~m}$ ( $\left.L_{\text {navires }}\right)$. La distance de sécurité entre les navires vaut $10 \%$ de cette longueur.

Dans les scénarios 2 et 4 , où le quai fonctionne de manière continue, il y a d'autres variables à considérer qui conditionnent les résultats de la relation entre $\varepsilon_{r}$ et $\rho_{L}$. En effet, quand les navires arrivent dans un port, selon la disposition des navires accostés au quai, ils peuvent rentrer dans des espaces différents. En fonction de l'espace choisi et de la position du navire dans cet espace où finalement ils sont disposés, les résultats peuvent différer.
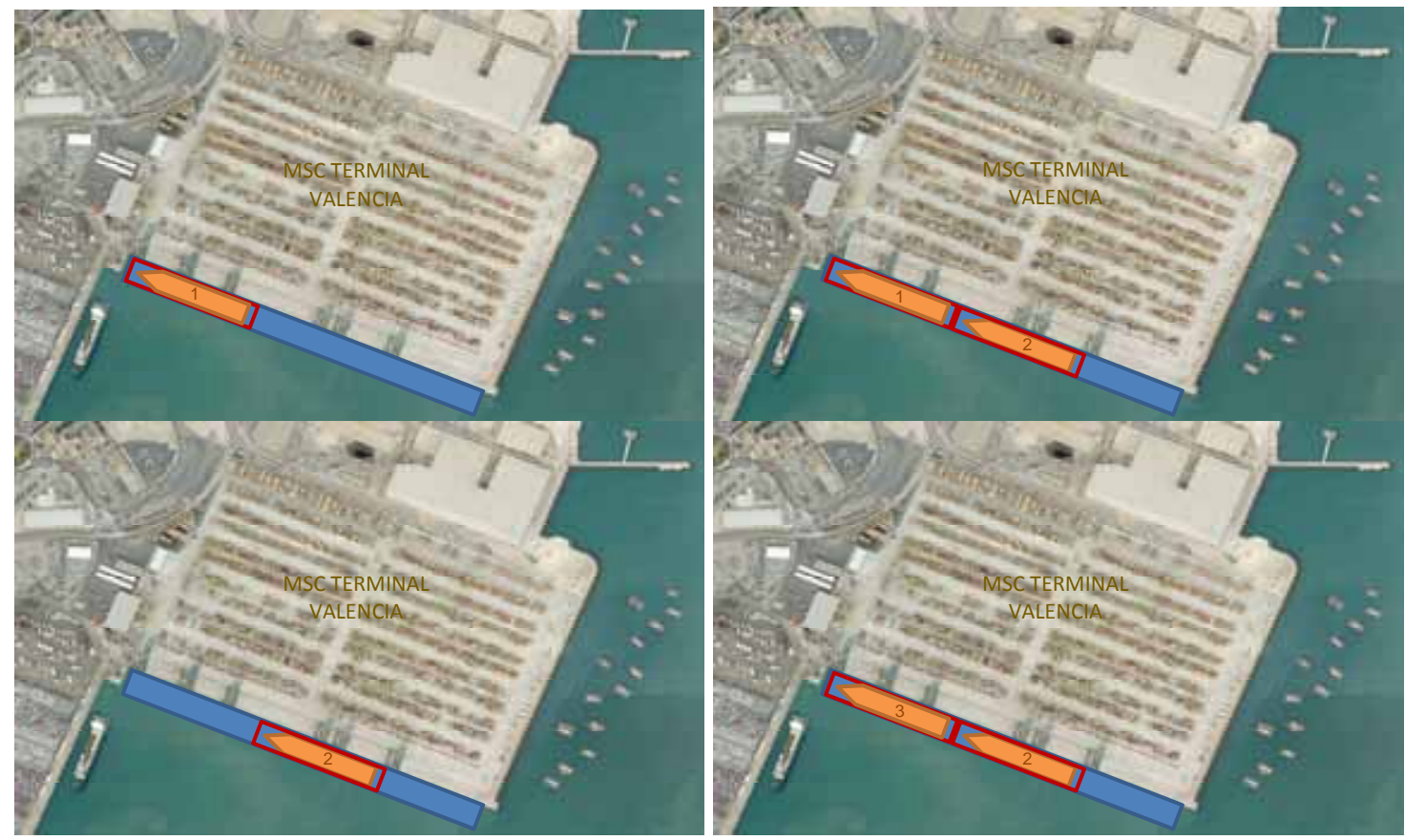

Figure 6. Routine d'assignation de poste d'accostage pour la formulation continue. 
Dans cette étude, on a supposé que lorsqu'un navire quitte le quai, tous les autre navires qui restent sont déplacés vers un côté du quai. De cette manière, quand un navire arrive au port, il n'y a qu'un seul espace pour le placer. En outre, on a supposé que dans cet espace, les navires sont accostés à côté des autres navires.

Il est évident que dans les terminaux, les navires ne sont pas déplacés chaque fois que l'un d'entre eux quitte le terminal (situation idéale). Néanmoins, les résultats de cette simulation sont des valeurs maximales qui serviront donc de référence à comparer à d'autres solutions. En outre, comme on a supposé une densité de probabilité des longueurs de navires constante, les résultats seront exactement les mêmes dans cette situation idéale qu'en supposant la routine d'assignation de poste d'accostage suivante : lors de l'arrivée du premier bateau au terminal, il est placé à un extrême ; lors des arrivées suivantes, les bateaux sont placés soit à côté de ceux qui sont déjà accostés (en prenant en compte la distance de sécurité), soit à la place de ceux qui partent (voir figure 6).

Il est important de remarquer que les deux formulations proposées dans cet article sont différentes mais appliquées à une même réalité. C'est-à-dire, même si une formulation considère le quai composé d'un nombre déterminé de postes d'accostage, et l'autre formulation considère le quai comme une ligne continue, les quais à conteneurs sont normalement exploités de manière continue. C'est pour cela que pour appliquer la formulation discrète il faut faire une translation et pour déterminer $N$ il faut appliquer l'équation (3). Cette équation peut donner un résultat non entier.

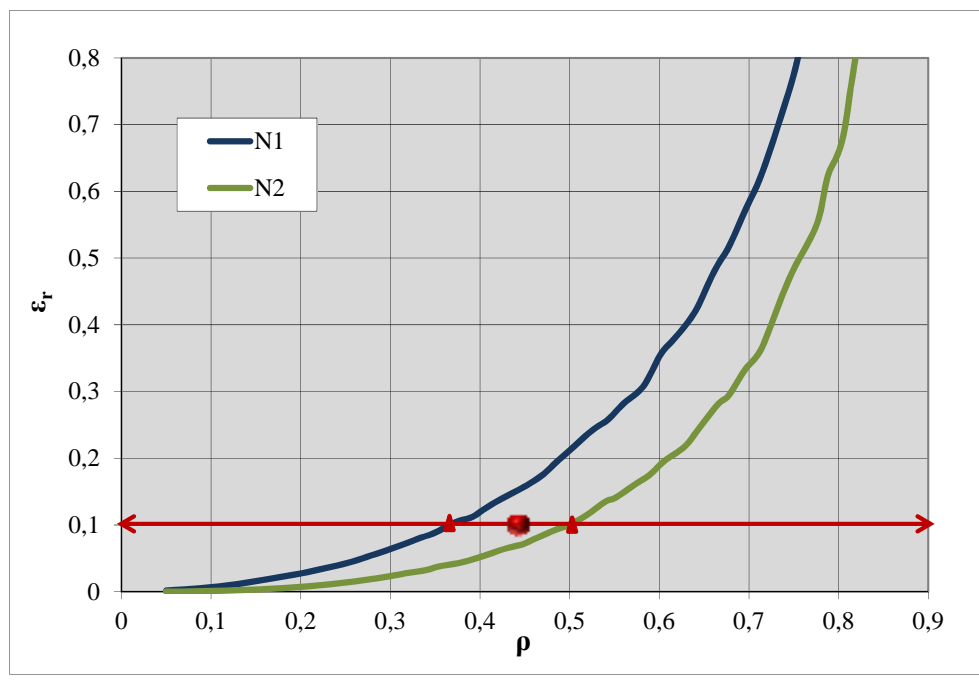

Figure 7. Interpolation de $\rho$ entre deux courbes de $N$ entier pour obtenir la valeur de $\rho$ quand $N$ est non entier.

Le fait que $N$ puisse ne pas être entier pose problème. La relation $\varepsilon_{r}-\rho$ est étudiée seulement quand $N$ est une valeur entière. Dans cette étude, afin de résoudre ce 
problème, on a interpolé les valeurs de $\rho$ entre les nombres entiers inférieur et supérieur de $N$ (voir figure 7).

Autant les fonctions de répartition $\mathrm{A}$ et $\mathrm{B}$ utilisées dans cette étude correspondent à la réalité du terminal MSCTV, autant, pour des raisons de confidentialité, les valeurs de la productivité $\left(P\right.$ ou $P_{L}$ ) ont été supposées par les auteurs. Pour les scénarios 1 et 3 , la productivité supposée par grue est de 23 mouvements à l'heure. En supposant deux grues par navire et des escales de 24h, dont 3h improductives, $P$ (productivité d'un poste d'accostage) atteint la valeur de 60,38 EVPs/(h·poste) (EVP/mouvement=1,5).

Pour les scénarios 2 et $4, P_{L}$ (productivité d'un mètre de quai) a été calculée comme $P$ fois le nombre de postes d'accostage divisé par L.

\section{Résultats}

Les résultats de la capacité pour chacun des quatre scénarios étudiés sont montrés dans le tableau 1.

Tableau 1. Résultats.

\begin{tabular}{lllll}
\hline & Scénario 1 & Scénario 2 & Scénario 3 & Scénario 4 \\
\hline Formulation & Discrète & Continue & Discrète & Continue \\
$L(m)$ & 656 & 656 & 756 & 756 \\
$L_{\text {navires }}(m)$ & 225 & 225 & 225 & 225 \\
$N$ & 2,65 & 2,65 & 3,05 & 3,05 \\
$\rho$ & 0,453 & - & 0,504 & - \\
$\rho_{L}$ & - & 0,275 & - & 0,494 \\
$P(E V P /(h \cdot p o s t e))$ & 60,38 & - & 60,38 & - \\
$P_{L}(E V P /(h \cdot m))$ & - & 0,24 & - & 0,24 \\
$H_{\text {an }}(h / a n)$ & $360 \times 24$ & $360 \times 24$ & $360 \times 24$ & $360 \times 24$ \\
\hline Capacité $(\mathbf{E V P / a n})$ & $\mathbf{6 2 6} \mathbf{0 6 2}$ & $\mathbf{3 7 9} \mathbf{9 7 8}$ & $\mathbf{8 0 2} \mathbf{3 7 9}$ & $\mathbf{7 8 6 ~ 3 4 6}$ \\
\hline
\end{tabular}

$N B$ : Les nombres en gris n'ont pas été utilisés directement pour calculer la capacité du scénario correspondant, mais ils sont vitaux afin de mieux comprendre les résultats.

En comparant les résultats du scénario 1 avec ceux du scénario 2, il apparait que l'estimation de la capacité peut grandement différer pour la même longueur du quai selon la formulation utilisée. En effet, l'estimation de la capacité en appliquant la formulation continue est inférieure de presque $40 \%$ par rapport à celle obtenue par la formulation discrète. La variable responsable de cette différence est le taux d'occupation qui passe de 0,453 à 0,275. L'explication réside dans la proportion entre $L$ et $L_{n a v i r e s}$, qui fait qu'une grande partie du quai est inoccupée en permanence (la partie décimale de $N$, ici 0,65 ) quand on applique la formulation continue. Comme la figure 8 le montre, quand $L=656 \mathrm{~m}$ et $L_{\text {navires }}=225 \mathrm{~m}, N=2,65$, c'est-à-dire, il n'y a que deux 
bateaux qui peuvent être accostés, et il y a une partie du quai qui est inoccupée en permanence $(0,65)$. Ce fait est seulement considéré lors de l'application d'une formulation continue, et donc, $\rho_{L}$ est très inférieur à $\rho$.

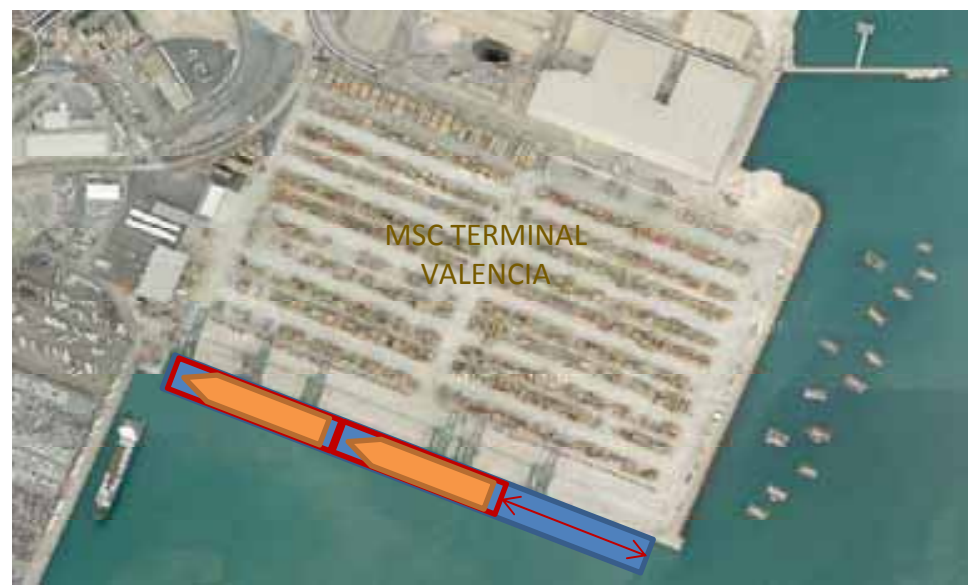

Figure 8. Quai de MSCTV avant son allongement conceptualisé comme une ligne continue.

Si on compare les résultats des deux formulations après l'allongement du quai, à savoir les scénarios 3 et 4, on remarque que même s'il existe une différence, celle-ci est très inférieure (2\%). En effet, en allongeant le quai la proportion entre $L$ et $L_{\text {navires }}$ (notée $N$ dans la formulation discrète) n'est pas si défavorable puisqu'on peut accoster trois bateaux de $225 \mathrm{~m}$ (voir figure 9) et il y a une très petite partie du quai inoccupée en permanence (la partie décimale de $N$ égale à 0,05 ). Cela se traduit par une valeur très supérieure de $\rho_{L}$, qui tend vers la valeur de $\rho$ du scénario 3, d'où le fait que la différence de capacité entre les scénarios 3 et 4 soit très réduite.

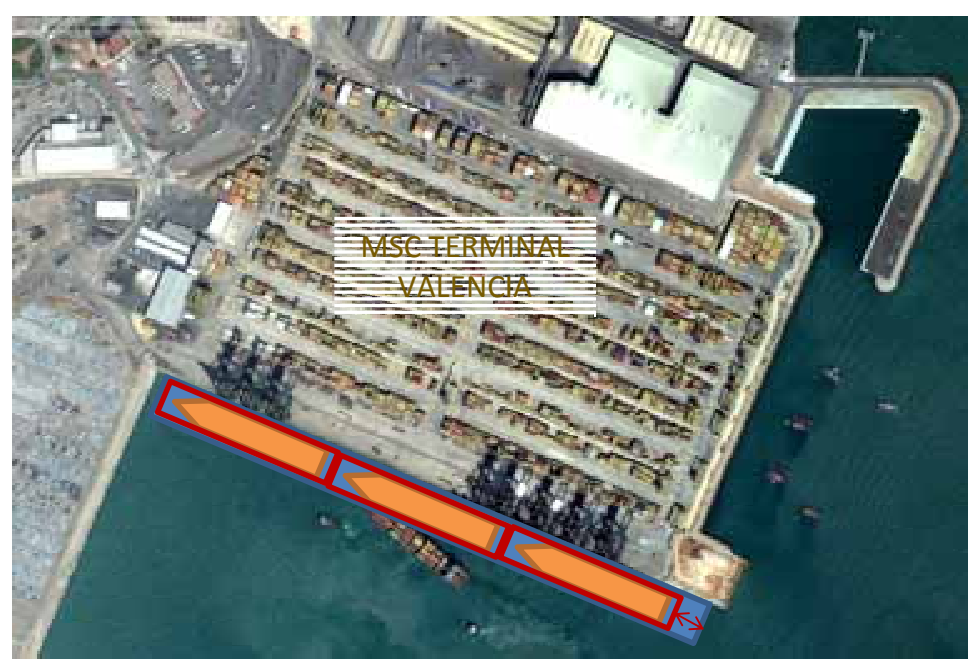

Figure 9. Quai de MSCTV après son allongement conceptualisé comme une ligne continue. 
Finalement, la comparaison des scénarios 2 et 4 permet de déduire qu'en allongeant le quai de $100 \mathrm{~m}$, à savoir 15\% approximativement, la capacité estimée en utilisant la formulation continue (qui représente plus fidèlement la réalité) est multipliée par plus de deux.

\section{Conclusions}

La principale conclusion qu'on peut tirer de cette étude est que lors de la planification des terminaux, il est nécessaire d'utiliser la formulation continue dans la mesure du possible. Pour cela, les outils de simulation sont requis, car autrement, on ne pourrait pas connaître la valeur de $\rho_{L}$.

Dans les cas où on ne peut pas utiliser des outils de simulation, et par conséquent, on utilise des formulations discrètes, il faut être conscient que les résultats peuvent surestimer la capacité réelle. Ceci est fonction de la proportion entre la longueur du quai et la longueur des navires (on peut considérer la longueur moyenne à laquelle on ajoute une distance de sécurité). Ainsi, plus la partie décimale de ce quotient est élevée, plus la capacité peut être surestimée.

De ce qui précède, on peut aussi conclure que, lors de la planification des terminaux, il est très important de connaître la longueur des navires qui vont y faire escale. Dans cette étude, afin de calculer les taux d'occupation, on a supposé que les fonctions de répartition des longueurs des navires sont constantes, car les planificateurs habituellement dimensionnent les quais pour une génération des navires déterminée : Panamax, post-Panamax, superpost-Panamax. Néanmoins, l'exploitation des quais doit faire face à des navires de longueurs différentes et l'influence que cette variation peut avoir sur le résultat du calcul du taux d'occupation d'un quai est évidente (pour la même longueur du quai, et les mêmes fonctions de répartition des arrivées et des services). Les constats précédents montrent qu'il est nécessaire de mener d'autres études sur le sujet afin de déterminer au mieux cette influence. On insiste sur le fait que pour bien continuer, les conclusions présentées dans cet article sont fondamentales.

\section{Références bibliographiques}

AGERSCHOU H. (2004). Chapter 1: Facilities Requirements. Planning and design of ports and marine terminals. Thomas Telford, $446 \mathrm{p}$.

AGUILAR J., OBRER-MARCO R. (2009). Consideraciones sobre la oferta y la demanda del servicio de atraque, en relación con la capacidad de las terminales de contenedores. X Jornadas Españolas de Costas y Puertos, Santander, pp 769-778.

DREWRY (2002). Global container terminals - Profit, performance and prospects. Drewry Shipping Consultants, Ltd. Royaume-Uni.

RODRIGUEZ F. (1977). Capacidad de los muelles. Ministerio de Obras Públicas, Madrid, $156 \mathrm{p}$. 